\title{
Metronomic chemotherapy for metastatic breast cancer to prolong time to treatment failure to 12 months or more
}

\author{
KEIICHI KONTANI ${ }^{1}$, SHIN-ICHIRO HASHIMOTO ${ }^{1}$, CHISA MURAZAWA $^{1}$, SHOKO NORIMURA ${ }^{2}$, \\ HIROAKI TANAKA ${ }^{3}$, MASAHIRO OHTANI ${ }^{4}$, NAOMI FUJIWARA-HONJO ${ }^{5}$, \\ MANABU DATE $^{6}$, HITOSHI HOUCHI ${ }^{3}$ and HIROYASU YOKOMISE ${ }^{1}$
}

\begin{abstract}
${ }^{1}$ Department of Respiratory, Breast and Endocrine Surgery, Kagawa University Faculty of Medicine, Kagawa University Hospital, Kita-gun, Kagawa 761-0793; ${ }^{2}$ Department of Surgery, Takamatsu Red Cross Hospital, Takamatsu, Kagawa 760-0017; ${ }^{3}$ Department of Pharmacy, Kagawa University Hospital, Kita-gun, Kagawa 761-0793; ${ }^{4}$ Kagawa Health Service Association, Health Care Center, Takamatsu, Kagawa 761-8071; ${ }^{5}$ Department of Radiology, Osaka Neurosurgery Hospital, Takamatsu 761-8083; ${ }^{6}$ Department of Surgery, Date Hospital, Takamatsu, Kagawa 760-0076, Japan
\end{abstract}

Received July 1, 2012; Accepted December 3, 2012

DOI: $10.3892 / \mathrm{mco} .2012 .49$

\begin{abstract}
The objective of treatment for metastatic breast cancer (MBC) is to control the disease or disease-related symptoms. Prolonged survival has also often been achieved by chemotherapeutic regimens in this setting. Long-term administration of one therapeutic regimen is essential for prolonging survival as well as for maintaining quality of life in these patients. In this study, we focused on time to treatment failure (TTF) as a parameter that predicts patient survival and we retrospectively compared clinical outcomes of patients with $\mathrm{MBC}$ who showed TTF of $\geq 12$ months (26 patients) and $<12$ months ( 29 patients). The proportion of hormone receptor-positive tumors and the number of prior chemotherapy regimens for MBC were significantly higher and tumor grade was lower in patients with TTF $\geq 12$ months compared to those with TTF $<12$ months. With regard to clinical outcomes, the objective response rate (ORR) in patients with $\mathrm{TTF} \geq 12$ months was significantly higher and median time to progression (TTP) and overall survival (OS) were longer compared to those with TTF $<12$ months. Of note, the proportion of patients who received metronomic regimens was significantly higher in patients with TTF $\geq 12$ months compared to those with TTF $<12$ months ( 80.8 vs. $24.1 \%$, $\mathrm{P}=0.00003)$. To assess the clinical benefit of metronomic regimens, the efficacy in patients receiving metronomic and those receiving non-metronomic regimens was compared.
\end{abstract}

Correspondence to: Professor Keiichi Kontani, Department of Respiratory, Breast and Endocrine Surgery, Kagawa University Faculty of Medicine, 1750-1 Miki-cho, Kita-gun, Kagawa 761-0793, Japan

E-mail: konbat@med.kagawa-u.ac.jp

Key words: metastatic breast cancer, metronomic chemotherapy, time to treatment failure
Although there was no difference in ORR between the two groups, median TTP and OS were significantly longer in the metronomic compared to the non-metronomic group (TTP: 30 vs. 4 months, $\mathrm{P}=0.0017$; OS: 68 vs. 28 months, $\mathrm{P}=0.0005$ ). The results suggested that metronomic chemotherapy is useful for palliative care and also improved clinical outcomes as a regimen for which long-term administration may be expected.

\section{Introduction}

As chemotherapy is used in adjuvant settings, anthracyclins and taxanes are included in standard regimens due to abundant evidence from several clinical trials over the past decades demonstrating a significant reduction in the risk of relapse or mortality of the disease (1-4). By contrast, there is no standard regimen recommended in metastatic settings. In most of the clinical trials for metastatic breast cancer (MBC), the efficacy of chemotherapeutic agents used as the first or second line of treatment for MBC was investigated (5-8). Anthracyclins have shown favorable activity that accounted for $30-50 \%$ of objective response rates (ORR) and $\geq 12$ months of overall survival (OS) when administered in the first-line regimen for MBC (6,7). Taxanes have shown efficacy consistent with that of anthracyclins in this setting (9-11). However, in the second or later line of treatment, many patients with MBC have already been treated with anthracyclins and taxanes in either adjuvant/neoadjuvant or metastatic settings. In these cases, alternative options should be considered. Results of clinical trials testing the efficacy and safety of vinorelbine, gemcitabine, TS-1, ixabepilone or capecitabine as treatment for MBC have been reported (9-20). Since these agents showed modest efficacy when administered alone, they have been recommended in combination with other cytotoxic agents. Taxanes were shown to have more benefit when used in combination with capecitabine, vinorelbine or gemcitabine compared with taxane monotherapy (21-25). Notably, although clinical trials assessing the efficacy of these agents, besides anthracyclins and taxanes, administered as monotherapy have shown an ORR 
of $0-20 \%$ and progression-free survival (PFS) of 2-5 months, these agents were expected to have favorable toxicity and survival of 8-12 months (9-20). Therefore, for palliative care, less toxic anticancer agents such as capecitabine, vinorelbine and gemcitabine are often recommended to be administered alone sequentially at the third or later line of treatment for MBC rather than administered concurrently (26).

Although the aim of treatment for MBC is control of the disease and disease-related symptoms, progression-free or disease-free long-term survival is often achieved by chemotherapy in patients with MBC. In these cases, the factors involved in clinical responses to the regimens used remain to be determined. In this study, we focused on time to treatment failure (TTF) as a parameter to compare clinical outcomes of the treated patients since prolonged TTF is thought to be correlated with long-term treatment with one therapeutic regimen, long time to progression (TTP) or OS with stable cancer status and good quality of life in treated patients. TTF is determined predominantly by a number of parameters that are predictable prior to treatment. Such parameters include regimen-related adverse events that are predictable in advance, patient agreement and physical and mental status, as well as the physician's confidence in the regimen and tumor responses to regimens that are unpredictable. In contrast to TTF, TTP, duration of response and OS are not predictable prior to treatment. Since TTF is the only parameter predictable in many cases prior to treatment and since TTF is closely correlated with the duration of treatment with one therapeutic regimen and survival, chemotherapy regimens that are expected to prolong TTF may be selected at the physician's discretion with consideration of patient status and prior chemotherapeutic regimens.

Metronomic chemotherapy is defined as continuous or frequent treatment of low doses of anticancer agents and is usually used as palliative care in patients who have been heavily pre-treated with cytotoxic drugs or who have poor performance status (PS) (27). Of note, metronomic chemotherapy used for palliation has been reported to result in favorable tumor responses and prolonged survival (28-30). The aim of this study was to determine whether prolonged TTF is correlated with a favorable clinical outcome and to determine which variable is responsible for the prolongation of TTF by comparing the clinicopathological characteristics and clinical outcomes between patients with $\mathrm{MBC}$ who showed TTF $\geq 12$ months and those who showed TTF $<12$ months. The clinical efficacy of metronomic regimens and that of non-metronomic regimens was also compared.

\section{Patients and methods}

Patients. In total, 55 patients with advanced or recurrent breast cancer who were treated with chemotherapeutic regimens at the Kagawa University Hospital between April, 2005 and December, 2011, were retrospectively analyzed for their clinicopathological characteristics and clinical outcomes. Mean age and number of pre-treated chemotherapeutic regimens for MBC of the patients were 58.11 (32 of 81) and 1.76 (0 of 4), respectively. Clinical outcomes examined in this study included TTF, defined as the duration from the initiation to the discontinuation of treatment; TTP, defined as the duration
Table I. Clinicopathological characteristics of patients with MBC.

\begin{tabular}{lccl}
\hline & TTF & TTF & \\
Characteristics & $\geq 12$ months & $<12$ months & P-value \\
\hline No. of patients & 26 & 29 & \\
Age (years) & $60.2 \pm 12.3$ & $56.3 \pm 12.6$ & 0.125 \\
DFI (months) & $62.2 \pm 50.4$ & $59.3 \pm 53.5$ & 0.19 \\
Line of CT for MBC & $2.2 \pm 1.31$ & $1.3 \pm 0.67$ & 0.003 \\
Prior adjuvant CT $(\%)$ & 61.1 & 78.9 & 0.242 \\
No. of metastatic sites & $1.9 \pm 0.86$ & $1.9 \pm 1.11$ & 0.96 \\
IDC (\%) & 94.1 & 100 & 0.395 \\
Tumor grade & $1.8 \pm 0.79$ & $2.6 \pm 0.67$ & 0.018 \\
HR-positive (\%) & 73.1 & 37.9 & 0.0096 \\
HER2-overexpressed (\%) & 34.8 & 17.9 & 0.172 \\
Triple negative $(\%)$ & 16 & 48.3 & 0.0129 \\
Metronomic CT $(\%)$ & 80.8 & 24.1 & 0.00003 \\
& & &
\end{tabular}

MBC, metastatic breast cancer; TTF, time to treatment failure; DFI, disease-free intervals; CT, computed tomography; IDC, invasive ductal carcinoma.

from initiation of treatment to disease progression or death from any cause; OS, defined as the duration from initiation of treatment to death from any cause; and ORR.

The patients were allocated the TTF $\geq 12$ months and TTF $<12$ months groups. Clinicopathological characteristics and outcomes were compared in the two groups (Table I).

Evaluation of therapeutic efficacy. Tumor responses were assessed by physical examination and computed tomography or magnetic resonance imaging, based on the Response Evaluation Criteria in Solid Tumors every 2-3 months during treatment and every 6 months following treatment. Complete response was defined as the absence of evidence of the disease, partial response was defined as a reduction in the product of the two largest perpendicular diameters of the target lesions by $<50 \%$, and progressive disease was defined as an increase in tumor size by $<25 \%$ or presence of a new lesion.

Statistical analysis. The Mann-Whitney U test or standard Chi-square procedures were used for comparison of the two groups. The effect of baseline characteristics, clinical responses or prognostic parameters on the risk of progression or death was calculated using the Kaplan-Meier method and the log-rank test of significance. A $95 \%$ confidence interval for the median of each variable was computed using the Brookmeyer and Crowley method. $\mathrm{P}<0.05$ was considered to indicate a statistically significant difference. P-values were two-sided. The SPSS statistical software system (SPSS Inc., Tokyo, Japan) was used for the calculations.

Ethical consideration. This study was in compliance with the guidelines of the Ethics Committee of the Kagawa University Hospital and conformed to the provisions of the Declaration 


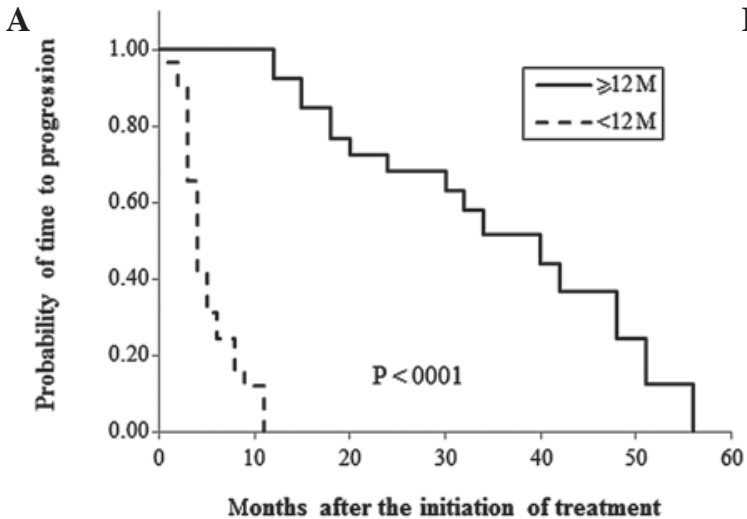

B

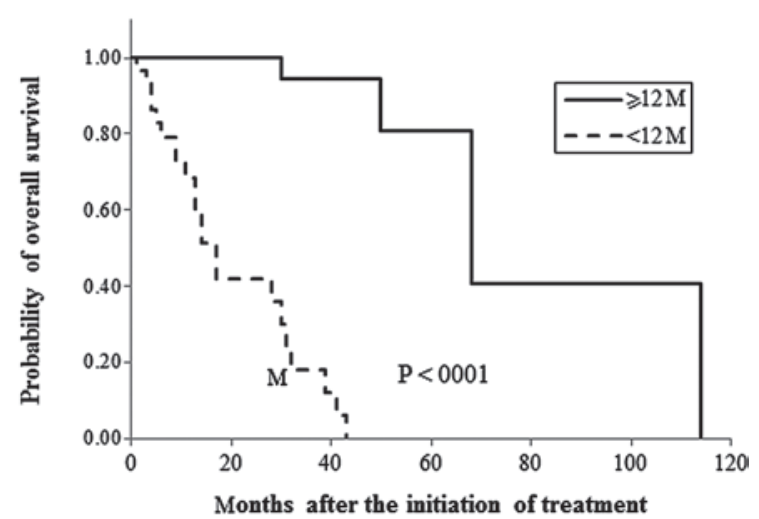

Figure 1. Comparison of survival rates of patients with time to treatment failure (TTF) of 12 months or more and patients with TTF of less than 12 months. Time to progression (TTP) and overall survival (OS) were compared between patients with TTF of 12 months or more and patients with TTF of less than 12 months in (A) and (B), respectively.

of Helsinki, 1995. Informed consent was obtained from all patients involved in the study.

\section{Results}

Comparison of clinicopathological characteristics and outcome between patients with TTF $\geq 12 \mathrm{M}$ and patients with $T T F<12 M$. There were 26 patients TTF $\geq 12$ months and 29 patients with TTF $<12$ months (Table I). Clinicopathological characteristics including age, disease-free interval defined as the interval from surgery for primary lesions to the diagnosis of cancer recurrence, number of prior chemotherapy regimens for $\mathrm{MBC}$, number of prior adjuvant chemotherapy regimens, number of metastatic sites, histologic types, tumor grade, hormone and HER2 status, as well as metronomic chemotherapy were compared in the two groups. Tumor grade and the proportion of triple negative cancers were significantly higher in the TTF $<12$ months group compared to the TTF $\geq 12$ months group. The proportion of hormone-sensitive breast cancers and the number of prior chemotherapy regimens for MBC were significantly higher in the TTF $\geq 12$ months group compared to the TTF $<12$ months group. Of note, the proportion of patients who received metronomic chemotherapy was significantly higher in the TTF $\geq 12$ months group compared to the TTF $<12$ months group (80.8 vs. $24.1 \%, \mathrm{P}=0.00003$ ).

Metronomic thermotherapy used in this study included capecitabine or TS-1 alone or in combination with oral cyclophosphamide and/or trastuzumab, which was used only for HER2-overexpressed cancer, or oral cyclophosphamide alone (data not shown). The metronomic chemotherapeutic regimens also included bisphosphonates or endocrine agents in certain cases with bone lesions or hormone-responsive cancer, respectively. Non-metronomic chemotherapy included paclitaxel, docetaxel or vinorelbine alone or in combination with capecitabine and/or trastuzumab, which was used only for HER2-overexpressed cancer, or anthracyclin-based regimens comprising cyclophosphamide, epirubucin and 5-fluorouracil. The non-metronomic therapeutic regimens included bisphosphonates in cases with bone lesions.

When clinical outcomes were compared in the two groups, ORR was significantly higher in the TTF $\geq 12$ months group
Table II. Comparison of clinical outcomes in patients with TTF of $\geq 12$ months or $<12$ months.

\begin{tabular}{|c|c|c|c|}
\hline $\begin{array}{l}\text { Clinical } \\
\text { outcomes }\end{array}$ & $\begin{array}{c}\text { TTF } \\
\geq 12 \text { months }\end{array}$ & $\begin{array}{c}\text { TTF } \\
<12 \text { months }\end{array}$ & P-value \\
\hline ORR $(\%)$ & 69.2 & 25 & 0.0009 \\
\hline $\begin{array}{l}\text { Median TTP } \\
\text { (months) }\end{array}$ & 40.0 & 4 & $<0.0001$ \\
\hline Median OS & 68.0 & 17 & $<0.0001$ \\
\hline
\end{tabular}

TTF, time to treatment failure; ORR, objective response rate; TTP, time to progression; OS, overall survival.

Table III. Reasons for selecting metronomic chemotherapy.

Reasons No. of patients

Heavy pretreatment 11

Patients' choice 9

Elderly or poor PS

Mental problem

Total 29

PS, performance status.

compared to the TTF $<12$ months group $(69.2$ vs. $25 \%, \mathrm{P}=0.0009$, Table II). Furthermore, patients in the TTF $\geq 12$ months group showed significantly longer median TTP and OS compared to patients in the TTF $<12$ months group (TTP: 40 vs. 4 months, $\mathrm{P}<0.0001$; OS: 68 vs. 17 months, $\mathrm{P}<0.0001$, Table II and Fig. 1).

Comparison of clinical outcomes in patients receiving metronomic regimens and patients receiving non-metronomic regimens. As described above, there was a statistically significant difference between the proportion of patients who received metronomic chemotherapy in the TTF $\geq 12$ months group and TTF $<12$ months groups. The 29 patients who received metronomic regimens included 11 patients who had 
Table IV. Comparison of clinicopathologic features in patients receiving metronomic regimens and those receiving non-metronomic regimens.

\begin{tabular}{lccc}
\hline Characteristics & Metronomic & Non-metronomic & P-value \\
\hline No. of patients & 28 & 27 & 0.0004 \\
Age (years) & $63.75 \pm 11.53$ & $52.26 \pm 10.80$ & 0.939 \\
DFI (months) & $49.20 \pm 48.51$ & $50.59 \pm 58.70$ & 0.056 \\
No. of prior CT for MBC & $2.03 \pm 1.20$ & $1.46 \pm 0.95$ & 0.144 \\
Prior adjuvant CT (\%) & 60 & 82.4 & 0.461 \\
Tumor grade & $2.14 \pm 0.86$ & $2.43 \pm 0.78$ & 0.185 \\
No. of metastatic sites & $1.71 \pm 0.81$ & $2.07 \pm 1.14$ & 0.417 \\
Hormone receptor $(\%)$ & 59.3 & 48.1 & 0.3 \\
HER2-overexpressed (\%) & 32 & 20 & 0.253 \\
Triple negative (\%) & 25.9 & 40.7 & \\
\hline
\end{tabular}

DFI, disease-free intervals; MBC, metastatic breast cancer; CT, computed tomography.

Table V. Comparison of clinical outcomes in patients receiving metronomic regimens and those receiving non-metronomic regimens.

\begin{tabular}{lccl}
\hline Outcomes & Metronomic & Non-metronomic & P-value \\
\hline $\begin{array}{l}\text { ORR (\%) } \\
\begin{array}{l}\text { Median TTF } \\
\text { (months) }\end{array}\end{array}$ & 50 & 42.3 & 0.575 \\
TTF & 75 & 4.0 & 0.00003 \\
$\geq 12$ months (\%) & & 18.5 & $<0.00001$ \\
$\begin{array}{l}\text { Median TTP } \\
\text { Median OS }\end{array}$ & 30 & 4.0 & 0.0017 \\
\hline
\end{tabular}

ORR, objective response rate; TTF, time to treatment failure; TTP, time to progression; OS, overall survival.

been heavily pre-treated, 9 patients who requested the regimen including 5 patients who were reluctant to experience hair loss, 7 patients who were elderly or had poor PS and 2 patients who had mental or psychiatric conditions (Table III). Clinical outcomes in patients receiving metronomic regimens and those receiving non-metronomic regimens were compared, since only the chemotherapeutic regimens listed in Table I could be selected by physicians prior to treatment. As shown in Table IV, none of the clinicopathological characteristics, with the exception of age, varied in the two groups. Mean age in the metronomic group was significantly higher compared to the non-metronomic group (63.75 vs. 52.26 years, $\mathrm{P}=0004$ ). Patients in the metronomic group showed a trend to receive more prior chemotherapy for $\mathrm{MBC}$ compared to patients in the non-metronomic group, although there was no statistically significant difference $(\mathrm{P}=0.056)$ (Table IV). The results suggested that metronomic regimens were indicated for elderly patients or patients who had been heavily pre-treated with chemotherapy. Median TTF, TTP and OS were significantly longer in the metronomic compared to the non-metronomic group (TTF: 18 vs. 4 months, $\mathrm{P}=0.00003$; TTP: 30 vs. 4 months, $\mathrm{P}=0.0017$; OS: 68 vs. 28 months, $\mathrm{P}=0.0005$, Table V). Furthermore, $75 \%$ of the patients who received metronomic regimens had $\geq 12$ months TTF, while only $18.5 \%$ of the patients who received non-metronomic regimens had $\geq 12$ months TTF (Table V). By contrast, no statistically significant difference was detected in ORR in the two groups.

\section{Discussion}

In this study, we retrospectively compared clinical outcomes in patients with MBC who had a long TTF ( $\geq 12$ months) and those who had a short TTF ( $<12$ months) to determine the factors predicting prognosis of patients with $\mathrm{MBC}$ who have received chemotherapy. The reason for selecting TTF for the comparison is that prolongation of the duration of one regimen is the most crucial characteristic for achieving long survival with minimized toxicity in treated patients. TTF is determined by tumor responses to the regimen used as well as by regimen-related adverse events, the patient's condition and agreement and the physician's confidence in the regimen for treatment of MBC. While tumor responses are unpredictable prior to treatment, possible regimen-related adverse events are predictable in several cases. Furthermore, using regimens that have abundant evidence concerning their efficacy and safety may be more readily accepted by patients and physicians. Thus, we thought that it is crucial to attempt to prolong the duration of one regimen, minimizing adverse events and maintaining satisfaction for both patients and physicians. Since ORR does not appear to correlate with survival, and TTP and OS are unpredictable prior to treatment, these parameters cannot be used for the predefined aim of treatment for MBC when selecting regimens $(10,31,32)$. Furthermore, a difference in OS has not been observed in several clinical trials comparing the efficacy of chemotherapeutic regimens.

For first-line chemotherapeutic regimens for MBC, anthracyclins have been recommended in chemonaive cases and taxanes in cases of prior treatment with anthracyclins in adjuvant settings. However, since anthracyclins and taxanes are standard chemotherapeutic agents in adjuvant settings, a 
number of patients with recurrent breast cancer have already been treated with regimens that included these agents. Anthracyclins and taxanes are also not indicated for patients with poor PS, insufficient bone marrow or organ function, or for elderly patients. Since patients with insufficient expression of the estrogen receptor (ER), i.e., $<50 \%$ of ER-positive cancer cells in the tumor or ER-positive/progesterone receptornegative tumors, are not expected to respond to endocrine therapy alone, chemotherapy is occasionally indicated in combination with endocrine therapy even in patients with hormone-sensitive tumors. For patients previously treated with anthracyclins or taxanes, therapeutic options are limited to less toxic agents such as vinorelbine, gemcitabine, capecitabine, TS-1 and ixabepilone. When vinorelbine, gemcitabine or liposomal doxorubicine was used alone for palliative care, ORR or PFS were reported to be modest, while OS was expected to be $\sim 12$ months (26). Capecitabine, approved by the Federal Diet and Food Association for administration in patients with MBC who were refractory to anthracyclins and taxanes, has shown favorable efficacy with ORR of $15-29 \%$, PFS of 3.1-4.9 months, and OS of 9.1-15 months in patients $(16,17,33)$. Therefore, capecitabine is strongly recommended for patients with MBC who have been heavily pre-treated, are elderly or have poor PS.

Metronomic chemotherapy is defined as 'continuous or frequent treatment with low doses of anticancer drugs, often given with other methods of therapy' in the dictionary of the 'Cancer Terms' of the National Cancer Institute. In this study, 29 of the 55 patients with MBC were selected to receive metronomic regimens. However, 20 of the 29 patients had mental or physical conditions, including insufficient bone marrow or organ function due to heavy prior chemotherapy or comorbidities, advanced age or poor PS. The remaining 9 patients requested this regimen, while 5 of them were reluctant to experience hair loss. Patients receiving metronomic regimens were older and had received more prior chemotherapy regimens compared to patients who received non-metronomic regimens (Table IV). A comparison of clinical outcomes in patients receiving metronomic regimens and patients receiving non-metronomic regimens showed that median TTF, TTP and OS were significantly prolonged in the metronomic group compared with those in the non-metronomic group (Table V). Seventy-five percent of patients in the metronomic group had $\geq 12$ months TTF, while $>80 \%$ of the patients in the non-metronomic group had $<12$ months TTF. The results showing no difference in ORR in the two groups suggest no correlation between ORR and survival.

In conclusion, metronomic chemotherapy for long-term administration is strongly recommended for patients with MBC who are elderly, have been heavily pre-treated with cytotoxic agents or have poor PS since it may lead to prolonging survival with minimized toxicity.

\section{Acknowledgements}

This study was supported in part by a Grant-in-Aid for Scientific Research from the Ministry of Education, Science, Sports and Culture, Japan (nos. 10671249, 13671380, 14571262 and 15591340).

\section{References}

1. Smith I and Chua S: Medical treatment of early breast cancer. I: adjuvant treatment. BMJ 332: 34-37, 2006.

2. Early Breast Cancer Trialists' Collaborative Group (EBCTCG): Effects of chemotherapy and hormonal therapy for early breast cancer on recurrence and 15-year survival: an overview of the randomized trials. Lancet 365: 1687-1717, 2005.

3. Bria E, Nistico C, Cuppone F, et al: Benefit of taxanes as adjuvant chemotherapy for early breast cancer: pooled analysis of 15,500 patients. Cancer 106: 2337-2344, 2006.

4. Buzdar AU, Singletary SE, Valero V, et al: Evaluation of paclitaxel in adjuvant chemotherapy for patients with operable breast cancer: preliminary data of a prospective randomized trial. Clin Cancer Res 8: 1073-1079, 2002.

5. Bontenbal M, Andersson M, Wildiers J, et al: Doxorubicin vs epirubicin, report of a second-line randomized phase II/III study in advanced breast cancer. EORTC Breast Cancer Cooperative Group. Br J Cancer 77: 2257-2263, 1998.

6. Jassem J, Pieńkowski T, Płuzańska A, et al: Doxorubicin and paclitaxel versus fluorouracil, doxorubicin, and cyclophosphamide as first-line therapy for women with metastatic breast cancer: final results of a randomized phase III multicenter trial. J Clin Oncol 19: 1707-1715, 2001.

7. Perez DJ, Harvey VJ, Robinson BA, et al: A randomized comparison of single-agent doxorubicin and epirubicin as first-line cytotoxic therapy in advanced breast cancer. J Clin Oncol 9: 2148-2152, 1991.

8. Bonneterre J, Dieras V, Tubiana-Hulin M, et al: Phase II multicentre randomised study of docetaxel plus epirubicin vs 5-fluorouracil plus epirubicin and cyclophosphamide in metastatic breast cancer. Br J Cancer 91: 1466-1471, 2004.

9. Sledge GW, Neuberg D, Bernardo P, Ingle JN, Martino S, Rowinsky EK and Wood WC: Phase III trial of doxorubicin, paclitaxel, and the combination of doxorubicin and paclitaxel as front-line chemotherapy for metastatic breast cancer: an intergroup trial (E1193). J Clin Oncol 21: 588-592, 2003.

10. Paridaens R, Biganzoli L, Bruning $\mathrm{P}$, et al: Paclitaxel versus doxorubicin as first-line single-agent chemotherapy for metastatic breast cancer: a European Organization for Research and Treatment of Cancer Randomized Study with cross-over. J Clin Oncol 18: 724-733, 2000.

11. Chan S, Friedrichs K, Noel D, et al: Prospective randomized trial of docetaxel versus doxorubicin in patients with metastatic breast cancer. J Clin Oncol 17: 2341-2354, 1999.

12. Rha SY, Moon YH, Jeung HC, et al: Gemcitabine monotherapy as salvage chemotherapy in heavily pretreated metastatic breast cancer. Breast Cancer Res Treat 90: 215-221, 2005.

13. Spielmann M, Llombart-Cussac A, Kalla S, et al: Single-agent gemcitabine is active in previously treated metastatic breast cancer. Oncology 60: 303-307, 2001.

14. Zelek L, Barthier S, Riofrio M, et al: Weekly vinorelbine is an effective palliative regimen after failure with anthracyclines and taxanes in metastatic breast carcinoma. Cancer 92: 2267-2272, 2001.

15. Gasparini G, Caffo O, Barni S, Frontini L, Testolin A, Guglielmi RB and Ambrosini G: Vinorelbine is an active antiproliferative agent in pretreated advanced breast cancer patients: a phase II study. J Clin Oncol 12: 2094-2101, 1994.

16. Blum JL, Dieras V, Lo Russo PM, Horton J, Rutman O, Buzdar A and Osterwalder B: Multicenter, Phase II study of capecitabine in taxane-pretreated metastatic breast carcinoma patients. Cancer 92: 1759-1768, 2001.

17. Blum JL, Jones SE, Buzdar AU, et al: Multicenter phase II study of capecitabine in paclitaxel-refractory metastatic breast cancer. J Clin Oncol 17: 485-493, 1999.

18. Jassem J, Carroll C, Ward SE, Simpson E and Hind D: The clinical efficacy of cytotoxic agents in locally advanced or metastatic breast cancer patients pretreated with an anthracycline and a taxane: a systematic review. Eur J Cancer 45: 2749-2758, 2009.

19. Pivot XB, Li RK, Thomas ES, et al: Activity of ixabepilone in oestrogen receptor-negative and oestrogen receptor-progesterone receptor-human epidermal growth factor receptor 2-negative metastatic breast cancer. Eur J Cancer 45: 2940-2946, 2009.

20. Ito Y, Osaki Y, Tokudome N, Sugihara T, Takahashi S, Iwase T and Hatake K: Efficacy of S-1 in heavily pretreated patients with metastatic breast cancer: cross-resistance to capecitabine. Breast Cancer 16: 126-131, 2009. 
21. Savio G, Laudani A, Leonardi V, Pepe A, Scianna C, Gebbia V and Agostara B: Treatment of metastatic breast cancer with vinorelbine and docetaxel. Am J Clin Oncol 29: 276-280, 2006.

22. Vici P, Di Lauro L, Sergi D, et al: A phase II trial of docetaxel and vinorelbine in patients with advanced breast cancer previously treated with anthracyclines. Oncology 75: 175-181, 2008.

23. Albain KS, Nag SM, Calderillo-Ruiz G, et al: Gemcitabine plus paclitaxel versus paclitaxel monotherapy in patients with metastatic breast cancer and prior anthracycline treatment. J Clin Oncol 26: 3950-3957, 2008.

24. Chan S, Romieu G, Huober J, et al: Phase III study of gemcitabine plus docetaxel compared with capecitabine plus docetaxel for anthracycline-pretreated patients with metastatic breast cancer. J Clin Oncol 27: 1753-1760, 2009

25. O'Shaughnessy J, Miles D, Vukelja S, et al: Superior survival with capecitabine plus docetaxel combination therapy in anthracycline-pretreated patients with advanced breast cancer: phase III trial results. J Clin Oncol 20: 2812-2823, 2002.

26. Oostendorp LJ, Stalmeier PF, Donders AR, van der Graaf WT and Ottevanger PB: Efficacy and safety of palliative chemotherapy for patients with advanced breast cancer pretreated with anthracyclines and taxanes: a systematic review. Lancet Oncol 12: 1053-1061, 2011.

27. Kerbel RS and Kamen BA: The anti-angiogenic basis of metronomic chemotherapy. Nat Rev Cancer 4: 423-436, 2004.

28. Colleoni M, Rocca A, Sandri MT, et al: Low-dose oral methotrexate and cyclophosphamide in metastatic breast cancer: antitumor activity and correlation with vascular endothelial growth factor levels. Ann Oncol 13: 73-80, 2002.
29. Colleoni M, Orlando L, Sanna G, et al: Metronomic low-dose oral cyclophosphamide and methotrexate plus or minus thalidomide in metastatic breast cancer: antitumor activity and biological effects. Ann Oncol 17: 232-238, 2006.

30. Wong NS, Buckman RA, Clemons M, et al: Phase I/II trial of metronomic chemotherapy with daily dalteparin and cyclophosphamide, twice-weekly methotrexate, and daily prednisone as therapy for metastatic breast cancer using vascular endothelial growth factor and soluble vascular endothelial growth factor receptor levels as markers of response. J Clin Oncol 28: 723-730, 2010

31. Nabholtz JM, Falkson C, Campos D, et al: Docetaxel and doxorubicin compared with doxorubicin and cyclophosphamide as first-line chemotherapy for metastatic breast cancer: results of a randomized, multicenter, phase III trial. J Clin Oncol 21: 968-975, 2003

32. Miller K, Wang M, Gralow J, et al: Paclitaxel plus bevacizumab versus paclitaxel alone for metastatic breast cancer. N Engl J Med 357: 2666-2676, 2007.

33. Fumoleau P, Largillier R, Clippe C, et al: Multicentre, phase II study evaluating capecitabine monotherapy in patients with anthracycline- and taxane-pretreated metastatic breast cancer. Eur J Cancer 40: 536-542, 2004. 